\title{
New Drug Regimen for Tuberculosis
}

\author{
Masoud Mardani ${ }^{1, *}$ \\ ${ }^{1}$ Infectious Diseases and Tropical Medicine Research Center, Shahid Beheshti University of Medical Sciences, Tehran, IR Iran \\ ${ }^{*}$ Corresponding author: Masoud Mardani, Infectious Diseases and Tropical Medicine Research Center, Shahid Beheshti University of Medical Sciences, Tehran, IR Iran. Tel: +98- \\ 2122439963, Fax:+98-2122439964, E-mail: masoud_mardani@ymail.com \\ Received: January 1, 2014; Accepted: January 5, 2014
}

Keywords:Tuberculosis, Multidrug-Resistant; Tuberculosis; Therapeutics

Tuberculosis (TB) remains a major global health problem, with 8.6 million cases and 1.3 million deaths in 2012 . The emergence of multidrug-resistant TB (MDR-TB) and extensively drug-resistant TB (XDR-TB) is an increasing global health problem. MDR-TB is widespread, with an estimated 450000 cases and 170000 deaths in 2012 (1), and XDR-TB was reported in 92 countries in 2012. Current TB drugs were developed over 40 years ago. However, curing TB requires long treatment duration and many TB drugs. Moreover, treatment of drug-resistant $\mathrm{TB}$ is difficult because less potent and more toxic drugs, as well as longer treatment duration are required. The lack of effective treatment could be one of the causes of low treatment success. For example, in 2010 , only $48 \%$ of MDR-TB patients were successfully treated (1). In the recent years, several promising TB drugs have been developed, and new regimens that combine these new drugs with existing drugs have been studied with the aim of reducing treatment duration and drug-drug interactions compared with previous regimens (2). Shortening of the treatment duration could yield a breakthrough in TB control, as the long duration of treatment leads many patients to default from treatment earlier than required. A randomized equivalence trial evaluating whether treatment duration could be shortened to a four-month regimen using standard doses of rifampicin in patients with a low risk of relapse (with noncavitary disease whose sputum cultures converted to show negative results after 2 months) showed significantly higher rates of relapse in the four-month arm than the standard six-month arm (3). This higher relapse rate in the shorter duration treatment arm may be due to the failure to eradicate dormant bacilli. Therefore, successful regimens that shorten the duration of treatment need to focus on introducing new drugs with novel mechanisms of action (3). In that sense, fluoroquinolones might have an important role. A recent randomized trial, showed higher relapse rate of $15 \%$ and $11 \%$ in the four-month thrice-weekly regimens of gatifloxacin or moxifloxacin with isoniazid, rifampin and pyrazinamide $\left(2 \mathrm{GHRZ}_{3} / 2 \mathrm{GHR}_{3}\right.$ or $\left.2 \mathrm{MHRZ}_{3} / 2 \mathrm{MHR}_{3}\right)$, compared with $6 \%$ in the standard six-month thrice-weekly treatment (2EHRZ3/4HR3) during the 24 months after the end of treatment (4). World Health Organization (WHO) guidelines on the treatment of MDR-TB recommend an intensive treatment phase of eight months and a total duration of 20 months in most patients (5). However, the results of such programmatic management of MDRTB have resulted unsatisfactory treatment success rates (6). An observational study that evaluated standardized treatment regimens for MDR-TB showed that they were highly effective with infrequent adverse outcomes; specifically, a regimen consisting of gatifloxacin in combination with ethambutol, pyrazinamide and clofazimine (five-month maintenance phase), supplemented with kanamycin, prothionamide and isoniazid during an intensive phase of four months or until sputum smear conversion (4KCGEHZP/5GEZC), achieved an $88 \%$ cure rate (7). Although there were significant limitations in this observational study, including a lack of comparable patients treated with the WHO recommended regimens, yet it is encouraging that this regimen successfully reduced the duration of MDR-TB treatment and increased the cure rate. Based on this report, the STREAM trial (The Evaluation of a Standardised Treatment Regimen of AntiTuberculosis Drugs for Patients with Multidrug-Resistant Tuberculosis; ISRCTN78372190) is now in progress, which is comparing the WHO-recommended individualized regimens with 4KCMEHZP/5MEZC (moxifloxacin instead of gatifloxacin). Fixed-dose combination (FDC) tablets, combining two or more anti-TB drugs, have been manufactured since the 1980s to increase patient compliance with anti-TB treatment; since FDCs were believed to prevent physician error in medication prescribing and patient error in selectively taking only one drug, they have been widely used (8). Many observational studies and clinical trials have been conducted to evaluate the effectiveness of FDCs. However, the results have not been consistent. Recently, a systemic review and meta-analysis was

Copyright (C) 2014, Infectious Diseases and Tropical Medicine Research Center. This is an open-access article distributed under the terms of the Creative Commons Attribution License, which permits unrestricted use, distribution, and reproduction in any medium, provided the original work is properly cited. 
performed to determine whether FDCs are effective in treating TB (9). At present, we are privileged to have anti TB combination therapy in Iran, which has led to increasing patient adherence, although some complications of combination therapy such as hepatotoxicity are considered as treatment limitations.

\section{References}

1. World Health Organization.. Global tuberculosis report 2013. Switzerland Geneva: WHO; 2013. Available from: http://www.who.int/ tb/publications/global_report/en/.

2. Zumla A, Nahid P, Cole ST. Advances in the development of new tuberculosis drugs and treatment regimens. Nat Rev Drug Discov. 2013;12(5):388-404.

3. Johnson JL, Hadad DJ, Dietze R, Maciel EL, Sewali B, Gitta P, et al. Shortening treatment in adults with noncavitary tuberculosis and 2-month culture conversion. Am J Respir Crit Care Med. 2009;180(6):558-63.

4. Jawahar MS, Banurekha VV, Paramasivan CN, Rahman F, Ram- achandran R, Venkatesan P, et al. Randomized clinical trial of thrice-weekly 4-month moxifloxacin or gatifloxacin containing regimens in the treatment of new sputum positive pulmonary tuberculosis patients. PLoS One. 2013;8(7).

5. Falzon D, Jaramillo E, Schunemann HJ, Arentz M, Bauer M, Bayona J, et al. WHO guidelines for the programmatic management of drug-resistant tuberculosis: 2011 update. Eur Respir J 2011;38(3):516-28.

6. Falzon D, Gandhi N, Migliori GB, Sotgiu G, Cox HS, Holtz TH, et al. Resistance to fluoroquinolones and second-line injectable drugs: impact on multidrug-resistant TB outcomes. Eur Respir J. 2013;42(1):156-68.

7. Van Deun A, Maug AK, Salim MA, Das PK, Sarker MR, Daru P, et al. Short, highly effective, and inexpensive standardized treatment of multidrug-resistant tuberculosis. Am J Respir Crit Care Med. 2010;182(5):684-92.

8. Blomberg B, Spinaci S, Fourie B, Laing R. The rationale for recommending fixed-dose combination tablets for treatment of tuberculosis. Bull World Health Organ. 2001;79(1):61-8.

9. Albanna AS, Smith BM, Cowan D, Menzies D. Fixed-dose combination antituberculosis therapy: a systematic review and metaanalysis. Eur Respir J. 2013;42(3):721-32. 